\title{
Longitudinal fracture analysis of inhomogeneous beams with continuously varying sizes of the cross-section along the beam length
}

\author{
Victor Rizov \\ Department of Technical Mechanics, University of Architecture, Civil Engineering and Geodesy, 1 Chr. Smirnensky blvd., 1046 \\ - Sofia, Bulgaria, E-mail: V_RIZOV_FHE@UACG.BG \\ Holm Altenbach \\ Lehrstubl für Technische Mechanik. und Geschäftsfübrender Leiter Institut für Mechanik G10/58, Fakultät für Maschinenbau, \\ Otto-von-Guericke-Universität Magdeburg, Universitätsplatz.2, 39106 Magdeburg, Deutschland, bolm.altenbach@ovgu.de
}

\begin{abstract}
Analyses of longitudinal fracture behavior of inhomogeneous beams which have continuously varying sizes of the cross-section along the beam length are carried-out. Beams of a rectangular cross-section are studied. It is assumed that beams exhibit continuous (smooth) material inhomogeneity along the width and height of the cross-section. A longitudinal crack located arbitrary along the beam height is analyzed. First, a cantilever beam with linearly varying width and height along the beam length is considered. The material of the beam has non-linear elastic mechanical behavior. The external loading consists of one bending moment applied at the free end of the lower crack arm. The fracture behavior is analyzed in terms of the strain energy release rate assuming that the modulus of elasticity is distributed continuously in the beam cross-section. The balance of the energy is considered in order to derive the strain energy release rate. A solution to the strain energy release rate is obtained also by considering the complementary strain energy for verification. The longitudinal fracture behavior of the inhomogeneous nonlinear elastic cantilever beam configuration is studied also for the cases when the variation of the width and height of the cross-section is described by sine and power laws.
\end{abstract}

KEYWORDS. Beam of varying cross-section; Longitudinal fracture; Material non-linearity; Inhomogeneous material

\section{OPEN $\bigcirc$ ACCESS \\ Citation: V. Rizov, H. Altenbach, Longitudinal fracture analysis of inhomogeneous beams with continuously varying sizes of the cross-section along the beam length, Frattura ed Integrità Strutturale, 53 (2020) 38-50.}

Received: 09.12.2019

Accepted: 20.04 .2020

Published: 01.07.2020

Copyright: (C) 2019 This is an open access article under the terms of the CC-BY 4.0 which permits unrestricted use, distribution, and reproduction in any medium, provided the original author and source are credited.

\section{INTRODUCTION} ne of the efficient ways to improve the stability and to increase the strength and load bearing capacity of the structures, and at the same time to reduce their weight is to use beams of continuously varying sizes of the cross-section along the beam length. Structural members and components with variable cross-section in the 
length direction are commonly used in various engineering applications in aeronautics, robotics, power stations, shipbuilding and car industry where the weight saving is of basic importance.

Beam structures with varying cross-section can be sophisticated further by using inhomogeneous materials. In contrast to the conventional homogeneous materials, such as metals, the material properties of inhomogeneous materials vary smoothly along one or more directions in the solid. Thus, the properties of inhomogeneous materials are continuous functions of spatial coordinates. The interest to the inhomogeneous materials is due mainly to the fact that certain kinds of inhomogeneous materials, such as functionally graded materials, have been widely used in aeronautical and mechanical engineering in the last thirty years [1 - 12]. The mechanical characteristics and microstructure of functionally graded materials can be tailored technologically during the manufacturing process in order to optimize the performance of the structural members and components to the external loadings and influences. The fracture behavior of inhomogeneous (functionally graded) structures and materials is of tremendous importance for practical engineering [13 - 15].

Various studies of the fracture behavior of functionally graded composite materials have been reviewed in [13]. Cracks oriented both parallel and perpendicular to the gradient direction have been analyzed by using methods of linear-elastic fracture mechanics. Fracture behavior under fatigue crack loading conditions has also been investigated. Rectilinear as well as curved cracks have been considered [13].

An approach for studying of delamination fracture behavior of a beam structure under creep loading conditions has been developed in [14]. The analysis has been carried-out assuming validity of the principles of linear-elastic fracture mechanics. An elevated temperature has been used to accelerate the delamination fracture at constant external loads. A double cantilever beam configuration has been examined. It has been found that the service lifetime can be successfully predicted by using a form of Paris law.

Various techniques for analyzing of functionally graded layers and sandwich beam constructions have been presented and discussed in [15]. Analyses of different beam configurations under static or dynamic loading conditions have been carriedout by using methods of linear-elastic fracture mechanics. Works on buckling behavior of sandwich constructions have also been reviewed. Static analyses of functionally graded beam structures resting on Pasternak elastic foundation have been reviewed too. Investigations of viscoelastic bending behavior of sandwich structures have been presented.

It should be noted that all of the above mentioned publications have been focused on fracture in beam configurations with constant cross-section along the beam length. Besides, linear-elastic behavior of the material has been assumed. Therefore, the aim of the present paper is to analyze the longitudinal fracture behavior of inhomogeneous beam configurations with constantly varying sizes (width and height) of the cross-section along the beam length. The analysis is carried-out assuming non-linear elastic mechanical behavior of the material. One of the motives for the present paper is the fact that certain kinds of inhomogeneous materials, such as functionally graded materials, can be built-up layer by layer $[11,12]$ which is a premise for appearance of longitudinal cracks between layers. It should be mentioned that the previous works of the author are concerned with longitudinal fracture analyses of inhomogeneous (functionally graded) beam configurations with constant sizes of the cross-section along the beam height [16 - 19]. The beam under consideration in the present paper exhibits smooth material inhomogeneity in both width and height directions. The longitudinal fracture is analyzed in terms of the total strain energy release rate by applying the theory for bending of prismatic beams since this theory can be used also for beams with varying cross-section along the beam length provided that the variation is not abrupt and the angle of inclination of the beam edge is small [20].

\section{THEORETICAL MODEL}

A $\mathrm{n}$ inhomogeneous non-linear elastic cantilever beam configuration with linearly varying cross-section along the beam length is shown in Fig. 1. The length of the beam is $l$. The beam is clamped in section $D$. The crosssection of the beam is a rectangle of width, $b$, and height, $b$. The variations of $b$ and $b$ along the beam length are given by the following linear laws:

$$
\begin{aligned}
& b=b_{n}+\frac{b_{t}-b_{n}}{l} x_{3}, \\
& b=b_{n}+\frac{b_{t}-b_{n}}{l} x_{3},
\end{aligned}
$$


where

$$
0 \leq x_{3} \leq l
$$

In (1) and (2), $b_{n}$ and $b_{n}$ are the width and height in the free end of the beam, the width and height in the clamping are denoted by $b_{t}$ and $b_{t}, x_{3}$ is the longitudinal centroidal axis of the beam (Fig. 1).

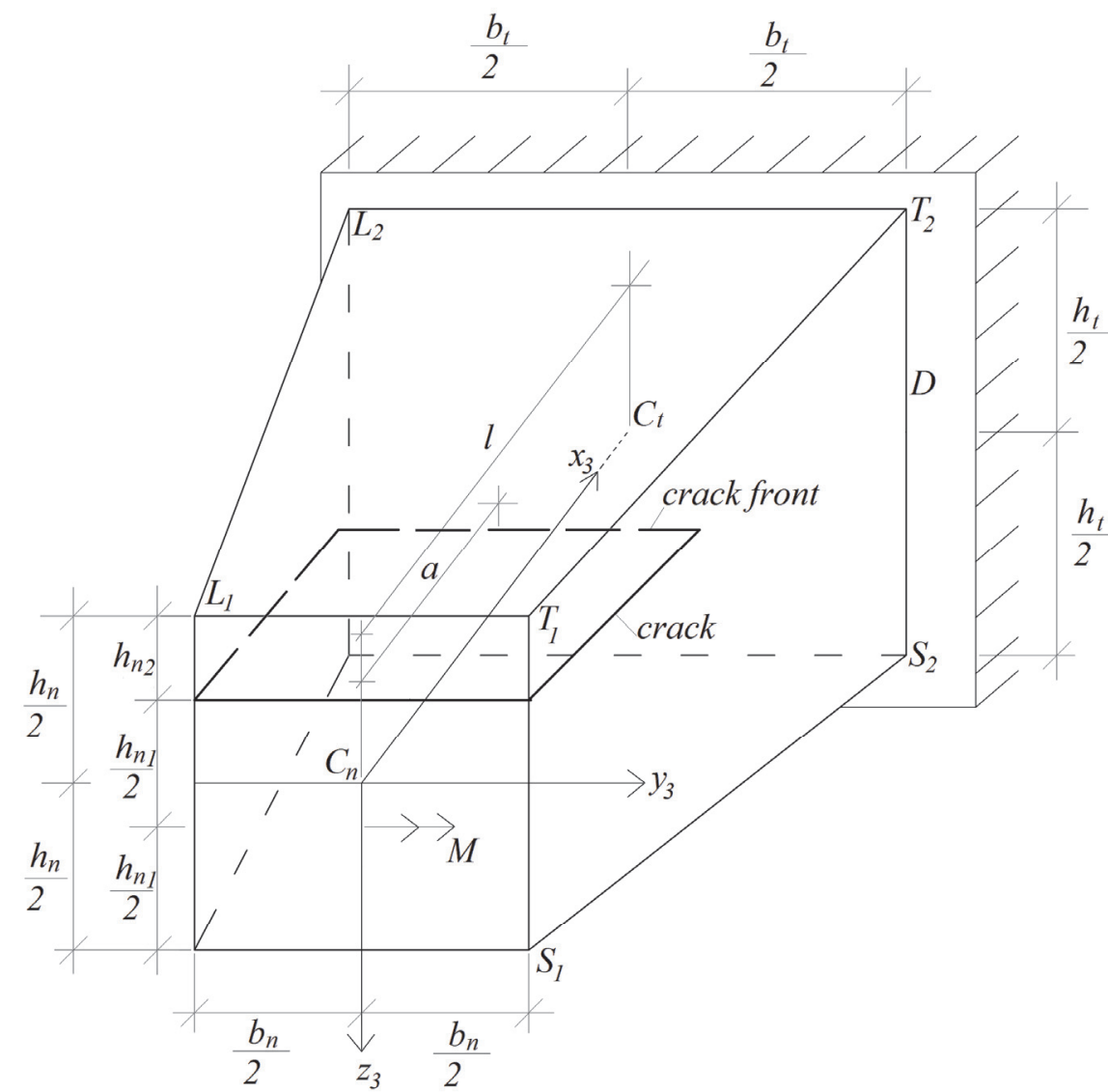

Figure 1: Geometry and loading of inhomogeneous cantilever beam with linearly varying sizes of the cross-section in the length direction.

The beam under consideration exhibits continuous (smooth) material inhomogeneity in both height and width directions of the cross-section. Thus, the distribution of the modulus of elasticity, $E$, in the beam cross-section is described by the following power law:

$$
E=\left(E_{T}-E_{L}\right)\left(\frac{y_{3}+\frac{b}{2}}{b}\right)^{g}+\left(E_{S}-E_{L}\right)\left(\frac{z_{3}}{b}+\frac{1}{2}\right)^{f}+E_{L},
$$

where

$$
\begin{aligned}
& -\frac{b}{2} \leq y_{3} \leq \frac{b}{2}, \\
& -\frac{b}{2} \leq z_{3} \leq \frac{b}{2} .
\end{aligned}
$$


In (4), $E_{L}, E_{T}$ and $E_{S}$ are, respectively, the values of the modulus of elasticity along the edges, $L_{1} L_{2}, T_{1} T_{2}$ and $S_{1} S_{2}$ of the beam (Fig. 1), $f$ and $g$ are material properties that control the material inhomogeneity in the height and width directions, respectively.

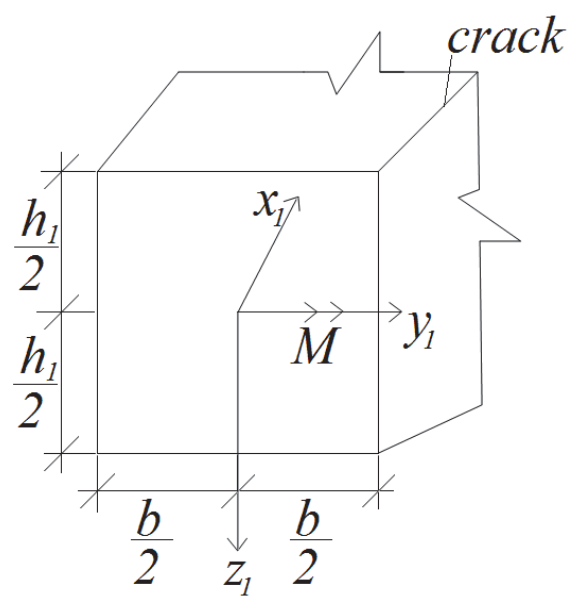

Figure 2: Cross-section of the lower crack arm.

A longitudinal crack of length, $a$, is located arbitrary along the beam height (Fig. 1). It should be noted that the present paper is motivated also by the fact that certain kinds of inhomogeneous materials, such as functionally graded materials, can be built-up layer by layer which is a premise for appearance of longitudinal crack between layers [8]. The thicknesses of the lower and upper crack arms in the free end of the beam are denoted by $b_{n 1}$ and $h_{n 2}$, respectively. The variations of the thicknesses of the lower and upper crack arms, $h_{1}$ and $h_{2}$, along the crack length are written as

$$
\begin{aligned}
& h_{1}=b_{n 1}+\frac{h_{t}-b_{n}}{2 l} x_{3}, \\
& h_{2}=b_{n 2}+\frac{h_{t}-b_{n}}{2 l} x_{3},
\end{aligned}
$$

where

$$
0 \leq x_{3} \leq a .
$$

The external loading of the beam consists of one bending moment, $M$, applied at the free end of the lower crack arm (Fig. 1). Thus, the upper crack arm is free of stresses. The longitudinal fracture behavior of the beam shown in Fig. 1 is studied in terms of the total strain energy release rate, $G$. For this purpose, the balance of the energy is analyzed. By assuming a small increase, $\delta a$, of the delamination crack length, the balance of the energy is expressed as

$$
M \delta \phi=\frac{\partial U}{\partial a} \delta a+G b \delta a
$$

where $\delta \phi$ the increase of the angle of rotation of the free end of lower crack arm, $U$ is the strain energy cumulated in the beam. From (10), the strain energy release rate is derived as

$$
G=\frac{M}{b} \frac{\partial \phi}{\partial a} \frac{1}{b} \frac{\partial U}{\partial a}
$$

The angle of rotation of the free end of lower crack arm is obtained by applying the Castigliano's theorem for structures exhibiting material non-linearity 


$$
\phi=\frac{\partial U^{*}}{\partial M}
$$

where $U^{*}$ is the complementary strain energy in the beam. Since the upper crack arm is free of stresses, the complementary strain energy is written as

$$
U^{*}=U_{1}^{*}+U_{2}^{*}
$$

where $U_{1}^{*}$ and $U_{2}^{*}$ are, respectively, the complementary strain energies cumulated in the lower crack arm and in the uncracked beam portion, $a \leq x_{3} \leq l$.

The complementary strain energy in the lower crack arm is expressed as

$$
U_{1}^{*}=\int_{0}^{a} \int_{-\frac{b}{2}}^{\frac{b}{2}} \int_{-\frac{b_{1}}{2}}^{\frac{b_{1}}{2}} u_{01}^{*} d x_{3} d y_{1} d z_{1}
$$

where $u_{01}^{*}$ is the strain energy density, $y_{1}$ and $z_{1}$ are the centroidal axes of the cross-section of the lower crack arm (Fig. 2).

The strain energy density is written as [16]

$$
u_{01}^{*}=\sigma \varepsilon-u_{01}
$$

where $\sigma$ is the normal stress, $\varepsilon$ is the strain, $u_{01}$ is the strain energy density in the lower crack arm. The mechanical behavior of the material is treated by the following stress-strain relation [21]:

$$
\sigma=E \varepsilon-H \varepsilon^{m},
$$

where $H$ and $m$ are material properties which describe the material non-linearity. The strain energy density is obtained by integrating of (16)

$$
u_{01}=\frac{E \varepsilon^{2}}{2}-\frac{H \varepsilon^{m+1}}{m+1} .
$$

By substituting of (16) and (17) in (15), one drives

$$
u_{01}^{*}=\frac{E \varepsilon^{2}}{2}-\frac{m H \varepsilon^{m+1}}{m+1} .
$$

By using (4), the distribution of the modulus of elasticity in the lower crack arm is written as

$$
E=\left(E_{T}-E_{L}\right)\left(\frac{y_{1}+\frac{b}{2}}{b}\right)^{g}+\left(E_{S}-E_{L}\right)\left(\frac{z_{1}}{b}-\frac{h_{1}}{2 h}+1\right)^{f}+E_{L},
$$

where

$$
-\frac{b}{2} \leq y_{1} \leq \frac{b}{2},
$$




$$
-\frac{h_{1}}{2} \leq z_{1} \leq \frac{h_{1}}{2}
$$

Since beams of high length to height ratio are under consideration in the present paper, the distribution of the strains in the cross-section of the lower crack arm is treated in accordance with the Bernoulli's hypothesis for the plane sections. Thus, $\varepsilon$ is expressed as

$$
\varepsilon=\varepsilon_{C_{1}}+\kappa_{y_{1}} y_{1}+\kappa_{z_{1}} z_{1},
$$

where $\varepsilon_{C_{1}}$ is the strain in the centre of the lower crack arm cross-section, $\kappa_{y_{1}}$ and $\kappa_{z_{1}}$ are the curvatures of lower crack arm in the $x_{1} y_{1}$ and $x_{1} z_{1}$ planes, respectively.

The curvatures of the lower crack arm and the strain in the centre of the cross-section are found from the equations for equilibrium of the elementary forces in the cross-section of the lower crack arm

$$
\begin{gathered}
N_{1}=\int_{-\frac{b_{1}}{2} \frac{b}{2}}^{\frac{b_{1}}{2}} \sigma d y_{1} d z_{1}, \\
M_{y_{1}}=\int_{-\frac{b}{2}}^{\frac{b}{2}} \int_{\frac{b}{2}}^{\frac{b_{1}}{2}} \sigma z_{1} d y_{1} d z_{1}, \\
M_{z_{1}}=\int_{-\frac{b_{1}}{2}}^{\frac{b}{2}} \int_{\frac{b}{2}}^{\frac{b_{1}}{2}} \sigma y_{1} d y_{1} d z_{1},
\end{gathered}
$$

where $N_{1}$ is the axial force, $M_{y_{1}}$ and $M_{z_{1}}$ are the bending moments with respect to the centroidal axes, $y_{1}$ and $z_{1}, \sigma$ is the normal stress, $b$ and $h_{1}$ are the width and height of the cross-section (Fig. 2). It is obvious that (Fig. 1)

$$
\begin{aligned}
& N_{1}=0, \\
& M_{y_{1}}=M, \\
& M_{z_{1}}=0 .
\end{aligned}
$$

After substituting of (16) in (23), (24) and (25) the equations for equilibrium are solved with respect to $\varepsilon_{C_{1}}, \kappa_{y_{1}}$ and $\kappa_{z_{1}}$ by using the MatLab computer program. Then $u_{01}^{*}$ is found by substituting of (19) and (22) in (18).

The complementary strain energy in the un-cracked beam portion is written as

$$
U_{2}^{*}=\int_{a}^{l} \int_{-\frac{b}{2}}^{\frac{b}{2}} \int_{-\frac{b}{2}}^{\frac{b}{2}} u_{02}^{*} d x_{3} d y_{2} d z_{2},
$$

where $u_{02}^{*}$ is the strain energy density, $y_{2}$ and $z_{2}$ are the centroidal axes. Formula (18) is used to obtain $u_{02}^{*}$. For this purpose, $\varepsilon$ is replaced with $\varepsilon_{d}$ where $\varepsilon_{d}$ is the distribution of the strains in the un-cracked beam portion. The 
distribution of $\varepsilon_{d}$ is found by replacing of $\varepsilon_{C_{1}}, \kappa_{y_{1}}$ and $\kappa_{z_{1}}$ with $\varepsilon_{C_{2}}, \kappa_{y_{2}}$ and $\kappa_{z_{2}}$ in (22) where $\varepsilon_{C_{2}}$ is the strain in the centre, $\kappa_{y_{2}}$ and $\kappa_{z_{2}}$ are the curvatures of the un-cracked beam portion. The strain in the centre and the curvatures are obtained by using the equations of equilibrium (23), (24) and (25). For this purpose, $b_{1}, \sigma, y_{1}$ and $z_{1}$ are replaced with $h, \sigma_{d}, y_{2}$ and $z_{2}$, respectively. The stresses, $\sigma_{d}$, is found by replacing of $\varepsilon$ with $\varepsilon_{d}$ in formula (16).

The strain energy cumulated in the beam is obtained as

$$
U=U_{1}+U_{2}
$$

where the strain energies in the lower crack arm and in the un-cracked beam portion are denoted by $U_{1}$ and $U_{2}$, respectively. Formulae (14) and (29) are used to determine $U_{1}$ and $U_{2}$. For this purpose, $u_{01}^{*}$ and $u_{02}^{*}$ are replaced with $u_{01}$ and $u_{02}$, respectively. The strain energy density in the un-cracked beam portion, $u_{02}$, is found by replacing of $\varepsilon$ with $\varepsilon_{d}$ in formula (17).

Finally, by substituting of $\phi$ and $U$ in (11), one obtains the following expression for the strain energy release rate:

$$
G=\frac{M}{b} \frac{\partial}{\partial M}\left(\int_{-\frac{b}{2}-\frac{b_{1}}{2}}^{\frac{b}{2}} \int_{01}^{\frac{b_{1}}{2}} u_{01}^{*} d y_{1} d z_{1}-\int_{-\frac{b}{2}-\frac{b}{2}}^{\frac{b}{2}} \int_{\frac{b}{2}}^{\frac{b}{2}} u_{02}^{*} d y_{2} d z_{2}\right)-\left(\int_{-\frac{b}{2}-\frac{b_{1}}{2}}^{\frac{b}{2}} \int_{01}^{\frac{b_{1}}{2}} u_{01} d y_{1} d z_{1}-\int_{-\frac{b}{2}-\frac{b}{2}}^{\frac{b}{2}} \int_{02}^{\frac{b}{2}} u_{02} d y_{2} d z_{2}\right)
$$

The integration in (31) is carried-out by using the MatLab computer program. MatLab is used also to determine the derivative, $\frac{\partial}{\partial M}(\ldots)$, in (31). It should be noted that $b, b, b_{1}, u_{01}, u_{02}, u_{01}^{*}$ and $u_{02}^{*}$ in (31) are obtained by (1), (2), (7), (17) and (18) at $x_{3}=a$.

The strain energy release rate is derived also by differentiating the complementary strain energy in the beam with respect to the crack are

$$
G=\frac{d U^{*}}{d A},
$$

where $d A$ is an elementary increase of the crack area. Since

$$
d A=b d a
$$

expression (32) takes the form

$$
G=\frac{d U^{*}}{b d a}
$$

where $d a$ is an elementary increase of the crack length.

By substituting of $U^{*}$ in (34), one obtains the following expression for the strain energy release rate:

$$
G=\frac{1}{b}\left(\int_{-\frac{b}{2}-\frac{b_{1}}{2}}^{\frac{b}{2}} \int_{01}^{\frac{b_{1}}{2}} u_{1}^{*} d z_{1}-\int_{-\frac{b}{2}-\frac{b}{2}}^{\frac{b}{2}} \int_{02}^{\frac{b}{2}} u_{02}^{*} d y_{2} d z_{2}\right)
$$

where $b, b, b_{1}, u_{01}^{*}$ and $u_{02}^{*}$ are found by (1), (2), (7), and (18) at $x_{3}=a$. The integration in (35) is performed by using the MatLab computer program. The fact that the strain energy release rate obtained by (35) is exact match of that 
found by (31) is an indication for correctness of the longitudinal fracture analysis of the inhomogeneous cantilever beam with linearly varying cross-section along the beam length carried-out in the present paper.

\section{CASE STUdies}

his section of the paper reports results which illustrate the influence of the varying cross-section of the beam in the length direction on the longitudinal fracture behavior. For this purpose, calculations of the strain energy release rate are performed by applying (31). The results obtained are presented in non-dimensional form by using the formula $G_{N}=G /\left(E_{L} b\right)$. The influence of the material inhomogeneity in the height and width directions, crack length, material non-linearity and the crack location along the beam height on the longitudinal fracture behavior are also analyzed. It is assumed that $b_{n}=0.008 \mathrm{~m}, h_{n}=0.006 \mathrm{~m}, l=0.130 \mathrm{~m}$, and $M=2 \mathrm{Nm}$.

The variation of the height of the cross-section along the beam length is characterized by $h_{t} / h_{n}$ ratio.

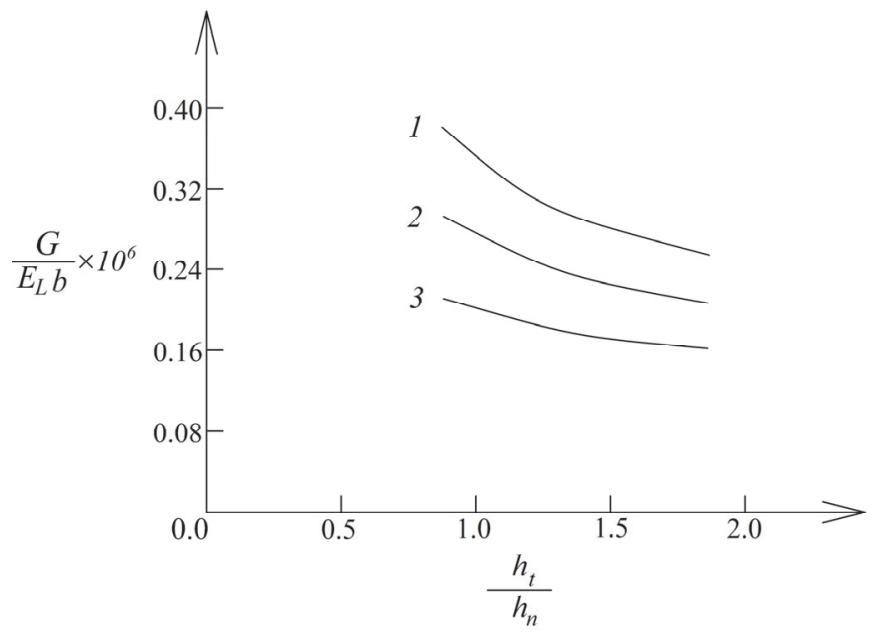

Figure 3: The strain energy release rate in non-dimensional form plotted against $h_{t} / h_{n}$ ratio (curve $1-$ at $E_{T} / E_{L}=0.5$, curve 2 - at $E_{T} / E_{L}=1.0$ and curve $3-$ at $\left.E_{T} / E_{L}=1.5\right)$.

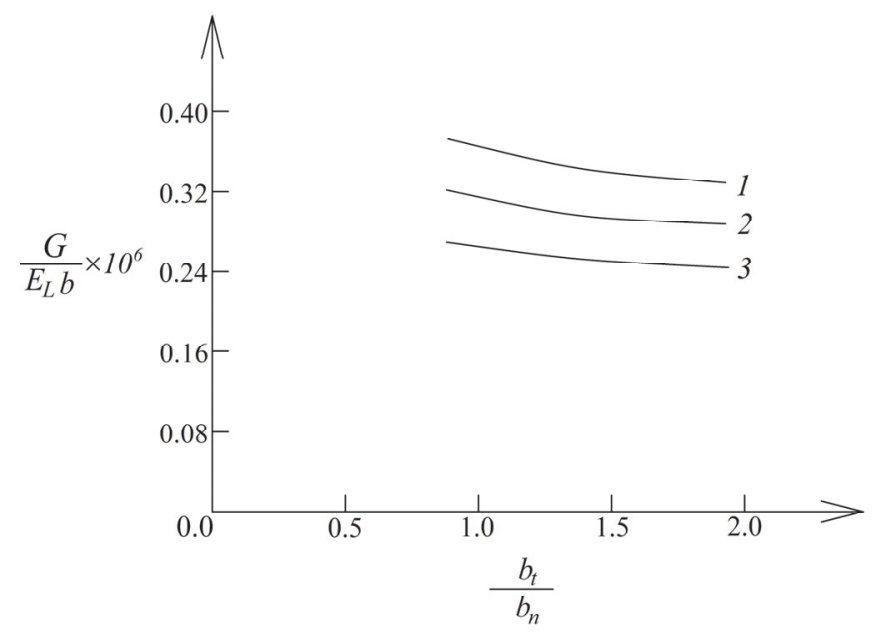

Figure 4: The strain energy release rate in non-dimensional form plotted against $b_{t} / b_{n}$ ratio (curve 1 - at $a / l=0.25$, curve $2-$ at $a / l=0.50$ and curve $3-$ at $a / l=0.75)$.

The influence of the variation of the height on the longitudinal fracture is illustrated in Fig. 3 where the strain energy release rate in non-dimensional form is plotted against $h_{t} / h_{n}$ ratio at three $E_{T} / E_{L}$ ratios (it should be mentioned that $E_{T} / E_{L}$ ratio characterizes the material inhomogeneity along the width of the cantilever beam configuration).

It is evident form Fig. 3 that the strain energy release rate decreases with increasing of $h_{t} / h_{n}$ ratio. The curves in Fig. 3 indicate also that increase of $E_{T} / E_{L}$ ratio leads also to decrease of the strain energy release rate.

The effect of the variation of the beam width in the length direction on the longitudinal fracture behavior is investigated too. The variation of the width is characterized by $b_{t} / b_{n}$ ratio. In order to evaluate the influence of the crack length on the longitudinal fracture, $a / l$ ratio is introduced.

One can get an idea of the influence the crack length in Fig. 4 where the strain energy release rate in non-dimensional form is plotted against $b_{t} / b_{n}$ ratio at three $a / l$ ratios. It can be observed in Fig. 4 that the strain energy release rate decreases with increasing of $b_{t} / b_{n}$ ratio. The strain energy release rate decreases also with increasing of $a / l$ ratio since the height and width of the beam cross-section increase towards the clamping.

The influence of the material inhomogeneity along the beam height on the longitudinal fracture behavior is analyzed. For this purpose, $E_{S} / E_{L}$ ratio is introduced. Calculations of the strain energy release rate are performed at various $E_{S} / E_{L}$ ratios. The results obtained are presented in Fig. 5 where the strain energy release rate in non-dimensional is plotted against $E_{S} / E_{L}$ ratio at three $h_{n 1} / h_{n}$ ratios. It can be observed in Fig. 5 that the strain energy release rate decreases with 
increasing of $E_{S} / E_{L}$ ratio. The crack location along the beam height is characterized by $h_{n 1} / h_{n}$ ratio. One can observe in Fig. 5 that the increase of $h_{n 1} / h_{n}$ ratio leads to decrease of the strain energy release rate.

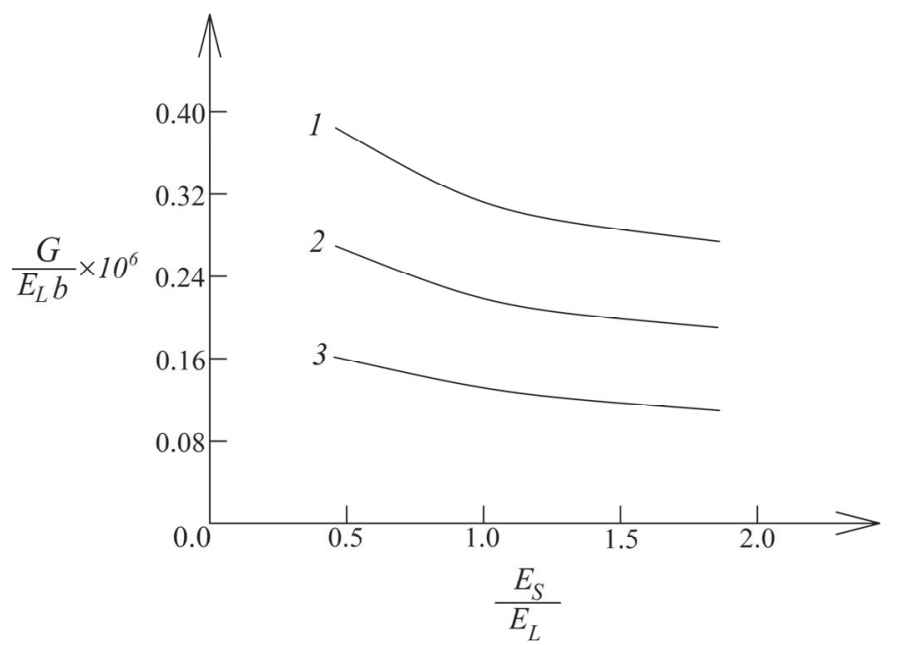

Figure 5: The strain energy release rate in non-dimensional form plotted against $E_{S} / E_{L}$ ratio (curve 1 - at $h_{n 1} / h_{n}=0.3$, curve $2-$ at $h_{n 1} / h_{n}=0.5$ and curve $3-$ at $h_{n 1} / h_{n}=0.7$ ).

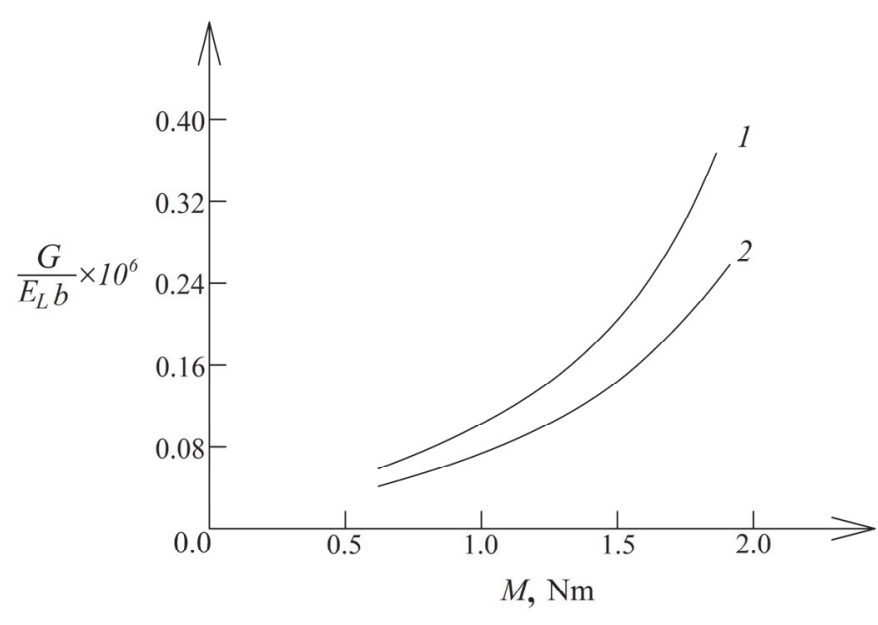

Figure 6: The strain energy release rate in non-dimensional form plotted against $M$ (curve 1 - at non-linear elastic behavior of the material and curve 2 - at linear-elastic behavior).

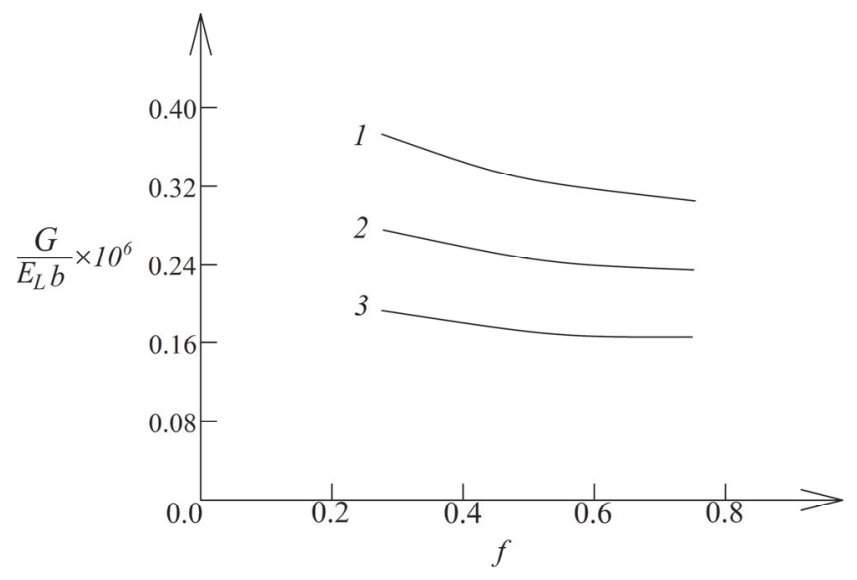

Figure 7: The strain energy release rate in non-dimensional form plotted against $f$ (curve $1-$ at $E_{S} / E_{T}=0.5$, curve $2-$ at $E_{S} / E_{T}=1.0$ and curve $3-$ at $\left.E_{S} / E_{T}=2.0\right)$.

The effect of the magnitude of the external loading of the beam on the longitudinal fracture behavior is studied also. For this purpose, calculations of the strain energy release rate are carried-out at various values of $M$. The calculated strain energy release rate in non-dimensional form is presented as a function of $M$ in Fig. 6. The curves in Fig. 6 show that the strain energy release rate quickly increases with increasing of $M$. The influence of the non-linear mechanical behavior of the material on the longitudinal fracture is investigated too. For this purpose, the strain energy release rate obtained assuming linear-elastic mechanical behavior of the inhomogeneous material is presented as a function of $M$ in Fig. 6. It should be noted that the linear-elastic solution to the strain energy release rate is derived by substituting of $H=0$ in formula (31) which follows from the fact that at $H=0$ the non-linear stress-strain relation (16) transforms into the Hooke's law where the modulus of elasticity of the inhomogeneous material is expressed by (4). It is evident from Fig. 6 that the non-linear mechanical behavior of the material causes increase of the strain energy release rate.

The influence of the material property $f$ on the longitudinal fracture behavior of the inhomogeneous non-linear elastic cantilever beam with linearly varying cross-section in the length direction is evaluated. The strain energy release rate in 
non-dimensional form is presented as a function of $f$ in Fig. 7 at three $E_{S} / E_{T}$ ratios. The curves in Fig. 7 indicate that the strain energy release rate decreases with increasing of $f$. One can observe also in Fig. 7 that the increase of $E_{S} / E_{T}$ ratio leads to decrease of the strain energy release rate.

It is interesting to investigate the effect of the law for variation of the sizes of rectangular cross-section along the beam length on the longitudinal fracture behavior. In order to elucidate this effect, a further two laws (sine and power) for continuous variation of the beam cross-section are considered. The variations of the width and height of the beam according to the sine law are written as

$$
\begin{aligned}
& b=b_{n}+\left(b_{t}-b_{n}\right) \sin \left(\frac{\pi x_{3}}{2 l}\right), \\
& b=b_{n}+\left(b_{t}-b_{n}\right) \sin \left(\frac{\pi x_{3}}{2 l}\right),
\end{aligned}
$$

where

$$
0 \leq x_{3} \leq l
$$

Formulae (36) and (37) indicate that the width and height vary smoothly from $b_{n}$ and $b_{n}$ at the free end of the beam to $b_{t}$ and $h_{t}$ at the clamped end of the beam. The variations of thicknesses of the lower and upper crack arms when the sine law is used are expressed as

$$
\begin{aligned}
& b_{1}=b_{n 1}+\frac{h_{t}-b_{n}}{2} \sin \left(\frac{\pi x_{3}}{2 l}\right), \\
& h_{2}=b_{n 2}+\frac{h_{t}-b_{n}}{2} \sin \left(\frac{\pi x_{3}}{2 l}\right),
\end{aligned}
$$

where

$$
0 \leq x_{3} \leq a
$$

When the power law is used, the variations of the width and height of the beam cross-section are written as

$$
\begin{aligned}
& b=b_{n}+\left(b_{t}-b_{n}\right)\left(\frac{x_{3}}{l}\right)^{\frac{3}{2}}, \\
& b=b_{n}+\left(b_{t}-b_{n}\right)\left(\frac{x_{3}}{l}\right)^{\frac{3}{2}}
\end{aligned}
$$

where

$$
0 \leq x_{3} \leq l
$$

Correspondingly, the variations of the thicknesses of the two crack arms are obtained as

$$
h_{1}=b_{n 1}+\frac{h_{t}-b_{n}}{2}\left(\frac{x_{3}}{l}\right)^{\frac{3}{2}} \text {, }
$$




$$
h_{2}=h_{n 2}+\frac{h_{t}-b_{n}}{2}\left(\frac{x_{3}}{l}\right)^{\frac{3}{2}} \text {, }
$$

where

$$
0 \leq x_{3} \leq a
$$

It is obvious from formulae (45) and (46) that the width and height vary smoothly from $b_{n}$ and $b_{n}$ at the free end of the beam to $b_{t}$ and $h_{t}$ at the clamped end of the beam.

The effect of the law for continuous variation of the beam cross-section on the longitudinal fracture behavior of the inhomogeneous non-linear elastic beam is illustrated in Fig. 8 where the strain energy release rate in non-dimensional form is presented as a function of the material property, $g$, for the three laws (linear, sine and power). It is evident from Fig. 8 that when the sine law is used the strain energy release rate is lower in comparison to that obtained at the linear law for variation of the width and height of the beam cross-section (this finding is explained by the fact that when the sine law is used the height and width of the beam cross-section are higher compared with the height and width according to the linear law).

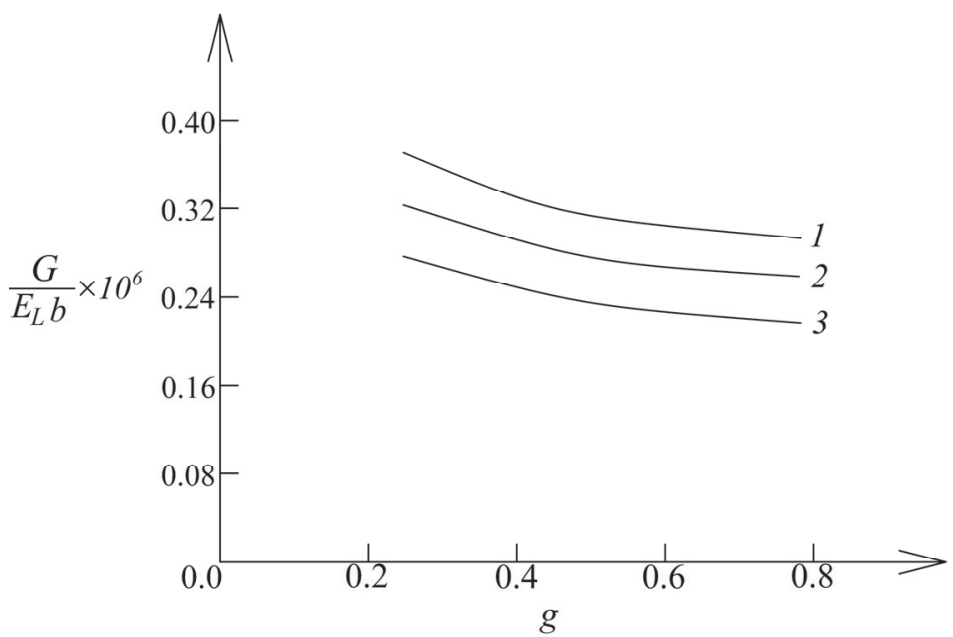

Figure 8: The strain energy release rate in non-dimensional form plotted against $g$ at three different laws for variation of the beam cross-section in the length direction (curve 1 - at power law, curve 2 - at linear law and curve 3 - at sine law).

The use of power law leads to obtaining of higher strain energy release rate compared to that calculated at linear law (this behavior is attributed to the lower sizes of the beam cross-section when the power law is used for describing the variation of the height and width along the beam length). It should be noted that when the sine and power laws are applied for describing the variation of the beam cross-section in the length direction, the strain energy release rate is obtained by (31). For this purpose, the sizes of the cross-section, $b, b$ and $b_{1}$, which are involved in (31) are calculated, respectively, by formulae by (36), (37) and (39) or by (42), (43) and (45). Concerning the effect of $g$, the curves in Fig. 8 show that the strain energy release rate decreases with increasing of $g$.

\section{CONCLUSIONS}

he main novelty of the present paper is that in contrast to previous papers [16 - 19] which deal with longitudinal fracture analysis of inhomogeneous beams with constant cross-section, the inhomogeneous beam considered here has continuously varying height and width in the length direction. The fracture is studied in terms of the total strain energy release rate assuming non-linear elastic mechanical behavior of the material. A solution to the strain energy release rate is derived by considering the balance of the energy. The strain energy release rate is obtained also by 
differentiating of the complementary strain energy in the beam with respect to the crack area for verification. The longitudinal fracture behavior is analyzed assuming continuous (smooth) material inhomogeneity in both height and width directions of the beam cross-section (the distribution of the modulus of elasticity in the cross-section is described by applying a power law). Special attention is paid to the influence of the continuously varying sizes of the beam crosssection on the longitudinal fracture behavior. It is found that the strain energy release rate decreases with increasing of $b_{t} / b_{n}$ and $b_{t} / b_{n}$ ratios (these ratios characterize the variation of the cross-section along the beam length). Concerning the effects of the crack length and the crack location along the beam height on the longitudinal fracture, the analysis reveals that the strain energy release rate decreases with increasing of $a / l$ and $h_{n 1} / h_{n}$ ratios. The decrease of the strain energy release rate with increasing of $a / l$ ratio is due to the fact that the sizes of the cross-section increase towards the clamped end of the beam. The longitudinal fracture behavior is studied also when the continuous variation of the height and width of the cross-section along the beam length is described by using sine and power laws. The investigation shows that when the sine law is used the strain energy release rate is lower in comparison to that derived when the sizes of the cross-section vary linearly along the beam length. When variation of the sizes is described by the power law the strain energy release rate is higher compared to that obtained by using linear law for describing the variation of beam crosssection. The research performed shows that the longitudinal fracture behavior of inhomogeneous beam structures can be controlled by using appropriate laws for continuous variation of the sizes of the beam cross-section in the length direction. The results obtained in the present paper could be useful in preliminary structural design of inhomogeneous beams with continuously varying cross-section in the cases when their longitudinal fracture behaviour is also required to be addressed.

\section{ACKNOWLEDGMENTS}

$\mathrm{R}$

izov is grateful for the financial support from the German Academic Exchange Service (DAAD) for his research stay in Department of Technical Mechanics, Institute of Mechanics, Otto-von-Guericke-University, Magdeburg, Germany.

\section{REFERENCES}

[1] Mortensen, A., Suresh, S., Functionally graded metals and metal-ceramic composites: Part 1 Processing, International Materials Review, 40 (1995) 239-265.

[2] Gasik, M.M., Functionally graded materials: bulk processing techniques, International Journal of Materials and Product Technology, 39 (1995) 20-29.

[3] Neubrand, A., Rödel, J., Gradient materials: An overview of a novel concept, Zeit f Met, 88 (1997) 358-371.

[4] Suresh, S., Mortensen, A., Fundamentals of functionally graded materials, IOM Communications Ltd, London (1998).

[5] Hirai, T., Chen, L., Recent and prospective development of functionally graded materials in Japan, Material Science Forum, 308-311 (1999) 509-514.

[6] Butcher, R.J., Rousseau, C.E., Tippur, H.V., A functionally graded particulate composite: Measurements and Failure Analysis, Acta Matererialia, 47 (1999) 259-268.

[7] Nemat-Allal, M.M., Ata, M.H., Bayoumi, M.R., Khair-Eldeen, W., Powder metallurgical fabrication and microstructural investigations of Aluminum/Steel functionally graded material, Materials Sciences and Applications, 2 (2011) 1708-1718.

[8] Saidi, H., Sahla, M., Vibration analysis of functionally graded plates with porosity composed of a mixture of Aluminum (Al) and Alumina (Al2O3) embedded in an elastic medium, Frattura ed Integrità Strutturale, 13 (2019) 286299.

[9] Chikh, A., Investigations in static response and free vibration of a functionally graded beam resting on elastic foundations, Frattura ed Integrità Strutturale, 14 (2019)115-126.

[10] Nagaral, M., Nayak, P. H., Srinivas, H. K., Auradi, V., Characterization and Tensile Fractography of Nano ZrO2 Reinforced Copper-Zinc Alloy Composites, Frattura ed Integrità Strutturale, 13 (2019) 370-376.

[11] Marae Djouda, J., Gallittelli , D., Zouaoui, M., Makke, A., Gardan , J., Recho, N., Crépin, J., Local scale fracture characterization of an advanced structured material manufactured by fused deposition modeling in 3D printing, Frattura ed Integrità Strutturale, 14 (2019) 534-540. 
[12] Bohidar, S.K., Sharma, R., Mishra, P.R., Functionally graded materials: A critical review, International Journal of Research, 1 (2014) 289-301.

[13] Tilbrook, M.T., Moon, R.J., Hoffman, M., Crack propagation in graded composites, Composite Science and Technology, 65 (2005) 201-220.

[14] Al-Khanbashi, A., Hamdy, A.E., Fracture mechanics approach to predict delamination lifetime in Mode II under constant loads, Journal of Adhesion Science and Technology, 18 (2004) 227-242.

[15] Sayyad, A.S., Ghugal, Y.M., Modeling and analysis of functionally graded sandwich beams: A review, Mechanics of Advanced Materials and Structures, 1 (2018) 1-20.

[16] Rizov, V.I., Non-linear elastic delamination of multilayered functionally graded beam, Multidiscipline Modeling in Materials and Structures, 13 (2017) 434-447.

[17] Rizov, V.I., Delamination analysis of a layered elastic-plastic beam, International Journal of Structural Integrity, 4 (2017) 516-529.

[18] Rizov, V.I., Analysis of cylindrical delamination cracks in multilayered functionally graded non-linear elastic circular shafts under combined loads, Frattura ed Integrità Strutturale, 46 (2018) 158-177.

[19] Rizov, V.I., Influence of material inhomogeneity and non-linear mechanical behavior of the material on delamination in multilayered beams, Frattura ed Integrità Strutturale, (2019) 468-481.

[20] Timoshenko, S., Strength of materials. Part II. Advanced theory and problems, Science (1965).

[21] Lukash, P.A., Fundamentals of non-linear structural mechanics, M. (1998). 